Problems in Freetown' by Dr. Milton Harvey and Professor J. C. Dewdney, Department of Geography, Fourah Bay College; 'The Government of Freetown' by Mr. Solomon Pratt, Practising Barrister and Economic Consultant, Freetown; "The Administration of the Law' by The Honourable Mr. Justice C. O. E. Cole, Acting Chief Justice of Sierra Leone; ' Religion in Freetown' by the Revd. E. W. Fashole-Luke, Department of Theology, Fourah Bay College. There was also a discussion led by Mr. George Tregson-Roberts, Chief Social Development Officer, Ministry of Social Welfare, on 'Social Problems of Freetown'.

All the papers presented at the symposium, together with one other paper- ${ }^{\text {"The }}$ Commercial Life of Freetown ' by Mr. John McKay, Department of Geography, Liverpool University-are to be published by the newly formed Sierra Leone University Press in association with Oxford University Press.

(Communicated by Mr. Michael Crowder, Director, Institute of African Studies)

\title{
Un Séminaire sur les Cultures Voltaïques
}

DEPurs plusieurs décenniés, divers ethnologues et historiens français ont mené des études intensives sur plusieurs populations de la Haute-Volta. Parallèlement, des chercheurs anglais ont poursuivi des enquetes ethnologiques dans le Nord du Ghana. A la suite de contacts pris, notamment au cours de congrès et colloques internationaux, il est apparu que des échanges réguliers entre ces deux groupes seraient fructueux pour l'un et pour l'autre et permettraient d'examiner les problèmes que pose l'étude de ces régions avec une efficacité accrue.

Une première réunion a eu lieu en France, groupant des spécialistes anglais et français, à Sonchamp (Seine et Oise), du 6 au 9 décembre 1965 . Pour cette première réunion, il avait été décidé d'un commun accord qu'aucun sujet particulier ne serait retenu, mais que chacun des participants exposerait l'état de ses rečherches. Les participants étaient les suivants: M. le Professeur M. Fortes, de l'Université de Cambridge, qui présidait les séances, a exposé des problèmes concernant les Tallensi; Madame G. Dieterlen, Directeur de Recherches au C.N.R.S., Directeur d'Études à l'E.P.H.E. (les Dogon et Kouroumba); Madame M. M. Schweeger-Hefel, Conservateur au Völkerkunde Museum de Vienna, et M. W. Staude, Attaché de Recherches au C.N.R.S. (les Kouroumba du Yatenga septentrional); Mrs. S. Drucker-Brown (les Mamprussi); Madame F. Izard, Chef de Travaux à l'E.P.H.E. (les Samo); M. J. Rouch, Maître de Recherches au C.N.R.S. (les Sonray et les migrations des Voltaîques au Ghana et en Côte d'Ivoire); M. le Professeur D. Zahan, Professeur à la Faculté des Lettres et des Sciences Humaines de Strasbourg, M. M. Izard, Attaché de Recherches au C.N.R.S., et M. R. Pageard, juriste et historien (les Mossi); M. G. le Moal, Attaché de Recherches au C.N.R.S. (les Bobo-Bing); M. M. Cartry, Attaché de Recherches au C.N.R.S. (les Gourmantche); M. M. Piault, Attaché de Recherches au C.N.R.S. (les Mauri). Les débats ont été entièrement enregistrés et seront très prochainement publiés dans la collection 'Recherches Voltaïques' (ronéo).

A la suite de ces premiers entretiens, le Professeur M. Fortes et nos collègues anglais nous ont invités à nous réunir à nouveau, pour rencontrer divers spécialistes qui n'avaient pu se rendre à Sonchamp, notamment M. le Professeur et Mme J. Goody de l'Université de Cambridge et M. J. Capron, Attaché de Recherches au C.N.R.S. Cette table ronde s'est tenue à Cambridge du 8 au I I décembre I966; l'enregistrement des débats donnera également lieu à une publication.

(Communication de Madame G. Dieterlen) 